\title{
Summer planetary-scale oscillations: aura MLS temperature compared with ground-based radar wind
}

\author{
C. E. Meek and A. H. Manson \\ Institute of Space and Atmospheric Studies, University of Saskatchewan, Saskatoon, S7N 5E2, Canada \\ Received: 8 December 2008 - Revised: 9 March 2009 - Accepted: 2 April 2009 - Published: 9 April 2009
}

\begin{abstract}
The advent of satellite based sampling brings with it the opportunity to examine virtually any part of the globe. Aura MLS mesospheric temperature data are analysed in a wavelet format for easy identification of possible planetary waves (PW) and aliases masquerading as PW. A calendar year, 2005, of eastward, stationary, and westward waves at a selected latitude is shown in separate panels for wave number range -3 to +3 for period range $8 \mathrm{~h}$ to 30 days (d). Such a wavelet analysis is made possible by Aura's continuous sampling at all latitudes $82^{\circ} \mathrm{S}-82^{\circ} \mathrm{N}$. The data presentation is suitable for examination of years of data. However this paper focuses on the striking feature of a "dish-shaped" upper limit to periods near $2 \mathrm{~d}$ in mid-summer, with longer periods appearing towards spring and fall, a feature also commonly seen in radar winds. The most probable cause is suggested to be filtering by the summer jet at $70-80 \mathrm{~km}$, the latter being available from ground based medium frequency radar (MFR). Classically, the phase velocity of a wave must be greater than that of the jet in order to propagate through it. As an attempt to directly relate satellite and ground based sampling, a PW event of period $8 \mathrm{~d}$ and wave number 2 , which appears to be the original rather than an alias, is compared with ground based radar wind data. An appendix discusses characteristics of satellite data aliases with regard to their periods and amplitudes.
\end{abstract}

Keywords. Atmospheric composition and structure (Instruments and techniques) - Meteorology and atmospheric dynamics (Middle atmosphere dynamics)

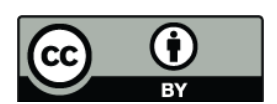

Correspondence to: C. E. Meek

(chris.meek@usask.ca)

\section{Introduction}

Many papers have been written in the past, and continue to be written, describing attempts to obtain characteristics of mesospheric planetary waves from a few isolated groundbased sites (Meek et al., 1996; Luo et al., 2002). Satellites now provide vast improvement in spatial sampling, but at the expense of temporal sampling. Though there have been a few satellites devoted to sampling upper atmospheric winds, e.g. UARS (Upper Atmosphere Research Satellite) and TIMED (Thermosphere Ionosphere Mesosphere Energetics and Dynamics), they have not replaced ground based sampling. On the other hand, temperature is commonly measured by satellites, e.g. Aura MLS (Mesosphere Limb Sounder), SABER (Sounding of the Atmosphere using Broadband Emission Radiometry) and others. A combination of satellite temperatures and ground based wind data may help to solve some temporal ambiguities (aliasing) in the former and spatial ambiguities due to sparse sampling in the latter.

Wavelet analysis is a convenient way to identify planetary wave events. Here, as in Manson et al. (2004a), the data are separated by wavenumber into apparent eastward, stationary, and westward propagating waves. As with any regularly sampled data, the potential for aliasing must be considered. Thus the frequency range is extended beyond the Nyquist period (which is $2 \mathrm{~d}$ here if single node sampling at a fixed location is considered) into the tidal period range, where globally coherent waves are also expected.

In the course of this work, a "dish shaped" feature was noted in PW frequency, an apparent frequency limit which increases from spring to mid summer (at which time the period is near $2 \mathrm{~d}$ ) and decreases towards fall, when slower waves, longer periods, are again seen. This feature has long been noted in radar data as well. It is suggested that it is due to filtering by the lower mesosphere zonal wind.

The paper is organized as follows:

Published by Copernicus Publications on behalf of the European Geosciences Union. 
The wavelet method as applied to Aura MLS satellite temperature data is described, and the year 2005 is presented for the latitude $51.7^{\circ} \mathrm{N}$. A short discussion of aliases and their amplitudes based on results derived in the appendix follows. The addition of peak MF radar winds below $80 \mathrm{~km}$ to the wavelet plot, represented as phase speed, leads to the suggestion that the dish-like appearance is caused by winds blocking upward progagating PW.

An apparent long period planetary wave in temperature with the unusual wavenumber of 2 is selected, checked for aliases, and compared with MF radar wind data at $40^{\circ}, 52^{\circ}$, and $70^{\circ} \mathrm{N}$.

\section{Data description}

High inclination orbit satellites have slow or no ("sun synchronous") precession in local time - basically the Earth turns under a completely or almost completely stationary orbit. The Aura satellite is sun synchronous, $\sim 90 \mathrm{~min}$ orbital period, with sampling $82^{\circ} \mathrm{S}$ to $82^{\circ} \mathrm{N}$. We use level 2, version $1.5 \mathrm{x}$, data here. Data are gridded in 121 latitude bins, approxmately 0.5 to $1^{\circ}$ steps depending on latitude, and 37 pressure levels, approximately 3 to $6 \mathrm{~km}$ steps depending on height. The line of sight is along the orbit, so the full latitude range is sampled all year. The stated orbital period means that tracks are spaced about $24^{\circ}$ in longitude at any latitude. As radar scientists we are used to thinking in terms of geometric height rather than pressure, so we translate the latter to approximate height by $\mathrm{h}(\mathrm{km})=(64.8 / 4$. $)\left(3 .-\log _{10} \mathrm{P}(\mathrm{mb})\right)$ in figure labels. At $90 \mathrm{~km}$, the averaging interval is about $15 \mathrm{~km}$ (Schwartz et al., 2008). Planetary waves usually have much longer vertical wavelengths, but we cannot rule out some minor reduction in amplitude due to height averaging.

The wind data are from MF radars located near Saskatoon $\left(52^{\circ} \mathrm{N}, 107^{\circ} \mathrm{W}\right)$, Platteville $\left(40^{\circ} \mathrm{N}, 105^{\circ} \mathrm{W}\right)$, and Troms $\varnothing$ $\left(70^{\circ} \mathrm{N}, 19^{\circ} \mathrm{E}\right)$ which operate continuously (e.g. Manson et al., 2003; Hall et al., 2003)).

\section{Analysis}

At a fixed height and latitude, a zonally propagating planetary wave is assumed to have the form

$v=V \cos (\omega t+m \ell-\phi)$

where $v$ is the measured parameter, $V$ is the wave amplitude, $\omega$ is the wave frequency, $m$ is the wavenumber, $t$ is time, and $\ell$ is the East longitude (radians). The phase $\phi$ can be interpreted as the time of maximum at zero longitude, or as the position of maximum (given by longitude $=\phi / \mathrm{m}$ ) at zero time.

Unlike satellites such as EP TOMS (Total Ozone Measuring System), the Aura MLS makes limb measurements on both ascending and descending nodes. Solar tides are expected to be significant at the upper mesospheric heights, and as will be discussed later, their non-linear interaction products can have aliases at the longer periods in which we are interested. Since Aura is in a sun synchronous orbit, its ascending (A) and descending (D) node samples are stationary with respect to migrating tides. These should appear as constant offsets to the A and D measurements at a particular latitude. If $\mathrm{A}$ and $\mathrm{D}$ means are separately subtracted, the solar migrating tides theoretically should be removed, but due to tidal variability there can be sidebands which remain as interesting residuals.

The Morlet wavelet amplitude (Goupillard et al., 1984) for temperature $\mathrm{T}$, frequency $\omega$ (period $=2 \pi / \omega)$ and wavenumber $m$ is calculated in the time window $t_{1}$ to $t_{2}$ (encompassing the A and D passes at times $t_{A_{1}}$ to $t_{A_{2}}$ and $t_{D_{1}}$ to $t_{D_{2}}$ which comprise $n_{A}$ and $n_{D}$ samples respectively) as

$$
2 W_{\text {corr }} \sqrt{X_{R}^{2}+X_{I}^{2}}
$$

where

$$
\begin{aligned}
X_{R}= & \frac{1}{2}\left\{\frac{1}{n_{A}} \sum_{t_{A}=t_{A_{1}}}^{t_{A_{2}}} W\left(T_{A}-\overline{T_{A}}\right) \cos \left(\omega t_{A}+m \ell_{A}\right)\right. \\
& \left.+\frac{1}{n_{D}} \sum_{t_{D}=t_{D_{1}}}^{t_{D_{2}}} W\left(T_{D}-\overline{T_{D}}\right) \cos \left(\omega t_{D}+m \ell_{D}\right)\right\} \\
X_{I}= & \frac{1}{2}\left\{\frac{1}{n_{A}} \sum_{t_{A}=t_{A_{1}}}^{t_{A_{2}}} W\left(T_{A}-\overline{T_{A}}\right) \sin \left(\omega t_{A}+m \ell_{A}\right)\right. \\
& \left.+\frac{1}{n_{D}} \sum_{t_{D}=t_{D_{1}}}^{t_{D_{2}}} W\left(T_{D}-\overline{T_{D}}\right) \sin \left(\omega t_{D}+m \ell_{D}\right)\right\} .
\end{aligned}
$$

This has the form of added A and D Fourier Transforms (FT, but at one frequency) where $W$ represents a truncated Gaussian window, amplitude 1.0 (centre) to 0.05 (ends), and $W_{\text {corr }}=2$ is the window correction factor. An advantage of the FT method over a least squares fit is that the resulting amplitudes are limited by the measured values. In the theoretical case of a single wave, both methods give the same result.

For each frequency the window length is arbitrarily chosen to be 6 times the period. The frequency range for the plot is divided into 600 pixels (logarithmic range covering periods $8 \mathrm{~h}-720 \mathrm{~h}$ ), and interval time (one year) into 800 pixels. There is no smoothing other than that inherent in the overlapping times and bandwidths of the windows.

Since Aura MLS data have very few gaps, only weak existence criteria are needed. We require that half the satellite passes covered by the windows must have data at the chosen latitude and height. If a window extends beyond the available data, no wavelet is calculated. The Nyquist period in Aura MLS data for zonal waves is $2 \mathrm{~d}$ for single node sampling, that is each location is sampled once per day per node, 
but here the plotted frequency range has been extended to much shorter periods in order to reveal, for example, nonmigrating tides (Meyer and Forbes, 1997) or their non-linear interaction byproducts, whose aliases might be mistaken for the long period waves of interest.

A $\log$ amplitude scale is used, "dB" $=20 \log _{10} T$, where $T$ is in $\mathrm{K}^{\circ}$, to enhance the appearance of the smaller oscillations at the expense of the larger, and the "gain" is also increased with spatial wavenumber since power tends to be related to geophysical spatial scales. For example " $+3 \mathrm{~dB}$ ' indicates an amplitude gain of $\sqrt{2}, "+6 \mathrm{~dB}$ " an amplitude gain of 2 etc. These act to bring weaker features into view. In addition data are clipped above a certain value - usually these extreme values occur at 24 or $12 \mathrm{~h}$ (which are aliases for stationary waves, as will be shown later).

Log frequency is a convenient way to expand the low frequency range. Both this and the log amplitude scales are helpful for a quick look but are somewhat detrimental when it comes to detecting aliases.

Figure 1 shows a wavelet plot for $2005,51^{\circ} \mathrm{N}, 91 \mathrm{~km}$. Three "cycles" are shown: infinity-24 h, 24-12 h, 12-8 h. As will be discussed in the next section, a peak in one cycle will appear again in this cycle as an alias and twice in each of the other two, but at different wavenumbers and usually with reduced amplitude.

A few comments on the high frequency (tidal) periods are pertinent here. Considering that the migrating tides should have been mostly removed, as evidenced by the null right at 24 and $12 \mathrm{~h}$ for wavenumbers +1 and +2 , respectively, it is not clear why the features near 24 and $12 \mathrm{~h}$ are so strong. If increasing significance criteria similar to that of Scargle (1982) are applied, a limit based on the ratio of spectral power at one frequency to total power, these are the last to survive. There are several possible reasons: (1) Amplitude modulation of tides, e.g. by transient PW, will have sidebands, not exactly at 24/12 h, which will remain; (2) Nonmigrating tides (NMT) are a possibility, but by definition these have different wavenumbers than the respective migrating, so the $12 \mathrm{~h}, m=+1$ and $24 \mathrm{~h}, m=+2$ are possible examples; (3) Because zero frequency is aliased to 24 and $12 \mathrm{~h}$, long term trends will also contribute, (4) The absolute bandwidth of a Fourier bin, $1 / \tau$, for a short sequence length $\tau$ is wider than for a long and so the shorter sequence has more background noise per bin, or put another way: the original variance is divided between Fourier bins so the fewer the number of bins, the more power each has. Further analyses with constant length sequences for all frequencies, linear frequency scales etc. (not shown) have suggested tidal modulation and aliases of very low frequencies and/or trends as the most probable cause.

The over-plotted line is calculated from Saskatoon peak zonal hourly wind near local noon (18:00-19:59 UT) in the height range $55-79 \mathrm{~km}$ (see Fig. 2). These daily values form a sequence which is averaged in parallel with the wavelet analysis, using the same frequency-dependent window and
Table 1. Amplitudes of aliases for orbit tilt $=8^{\circ}, t_{s}=1 \mathrm{~d}$, $t_{\text {orb }}=91.6791 \mathrm{~min}$ versus latitude.

\begin{tabular}{rrrrrrr}
\hline & \multicolumn{7}{c}{ Alias order } \\
N.Lat. $\left(^{\circ}\right)$ & 0 & 1 & 2 & 3 & 4 & 5 \\
\hline 0.0 & 1.00 & 0.00 & 1.00 & 0.00 & 1.00 & 0.00 \\
10.0 & 1.00 & 0.02 & 1.00 & 0.07 & 1.00 & 0.12 \\
20.0 & 1.00 & 0.05 & 0.99 & 0.15 & 0.98 & 0.25 \\
30.0 & 1.00 & 0.08 & 0.99 & 0.24 & 0.95 & 0.40 \\
40.0 & 1.00 & 0.12 & 0.97 & 0.35 & 0.89 & 0.56 \\
50.0 & 1.00 & 0.17 & 0.94 & 0.48 & 0.78 & 0.75 \\
60.0 & 1.00 & 0.24 & 0.88 & 0.67 & 0.55 & 0.94 \\
70.0 & 1.00 & 0.39 & 0.70 & 0.93 & 0.01 & 0.92 \\
80.0 & 1.00 & 0.80 & 0.27 & 0.37 & 0.85 & 0.99 \\
\hline
\end{tabular}

correction factor that was applied to temperature. Finally this average wind speed and the global wavenumber for the plot, are converted to a frequency. That is, a wave of this frequency and wavenumber will have a (westward) phase speed equal to the radar wind. A black dot is placed in the plot if this frequency, converted to a frequency pixel index, coincides with that of the row being plotted.

\section{Aliasing}

Before we can have confidence that we are looking at a PW signature, we must make sure it is not just an alias of a higher frequency event. Salby (1982) has given a very mathematical description of aliasing in satellite data analysis. In the appendix we present what seems to us a simpler derivation, leading to the results we need. These are the following. If an actual wave has wavenumber $m_{1}$ and frequency $f_{1}$ then its aliases are $m, f$, given by

$m=m_{1}+n, \quad$ and $\quad \frac{f}{f_{s}}=\frac{f_{1}}{f_{s}}+n$

where $f_{s}$ is $1 / t_{s}$ where $t_{s}$ is the time for the satellite to cover all longitudes (e.g. $1 \mathrm{~d}$ for Aura), $m, m_{1}$ must be integers, and $n=0, \pm 1, \pm 2, \pm 3 \ldots$

The amplitude of an alias is given by

$V_{A \& D}=V_{0} \cos \frac{n}{2} \Delta L$

where $V_{0}$ is the amplitude of the actual wave $(n=0), \mathrm{n}$ is the "alias order": $0, \pm 1, \pm 2, \ldots$, and $\Delta L$ is the azimuthal angle between ascending (A) and descending (D) node samples at the same latitude in the sun synchronous reference system.

Table 1 shows some values for a satellite with sampling parameters similar to Aura. SABER is somewhat different in that it looks perpendicular to its orbit path, and changes sides every 2 months, so as not to be looking towards the sun. Since the sampled path is not an earth-centred circle, $\Delta L$ must be calculated for the current orbit configuration. 


\section{AuraMLS Temperatures: $0.00215 \mathrm{mb}(\sim 92 \mathrm{Km})$, Latitude $52 \mathrm{~N}$.}

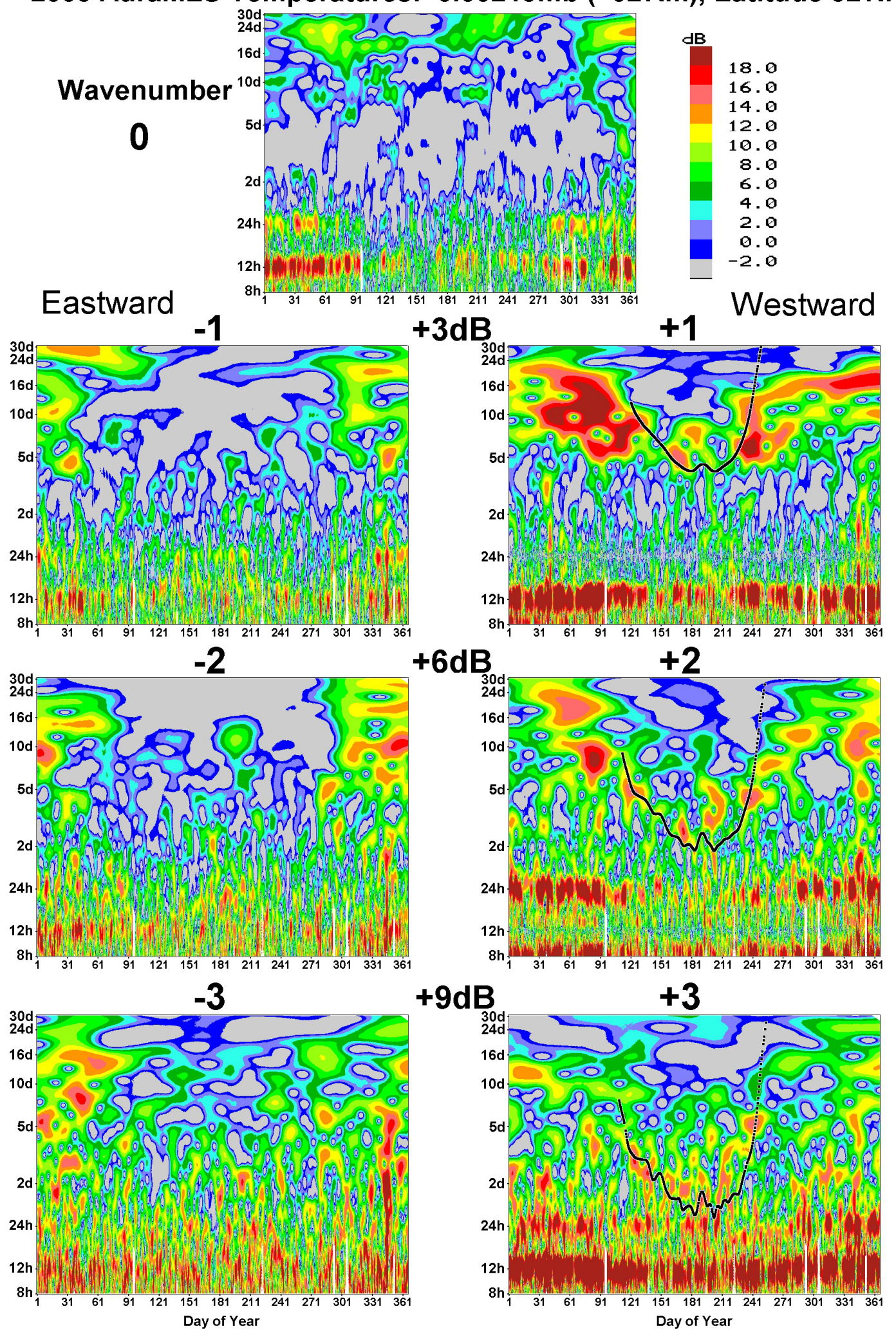

Fig. 1. 2005 Aura MLS temperature wavelets for wavenumbers 0 to \pm 3 . The sequences are 6 periods long, windowed with a Gaussian. Some clipping of strong signals (e.g. at $12 \mathrm{~h}$ ) has been allowed. The "gain" of the plot is increased with wave number. The overplotted line is a phase speed limit estimated from radar data. (see text). 


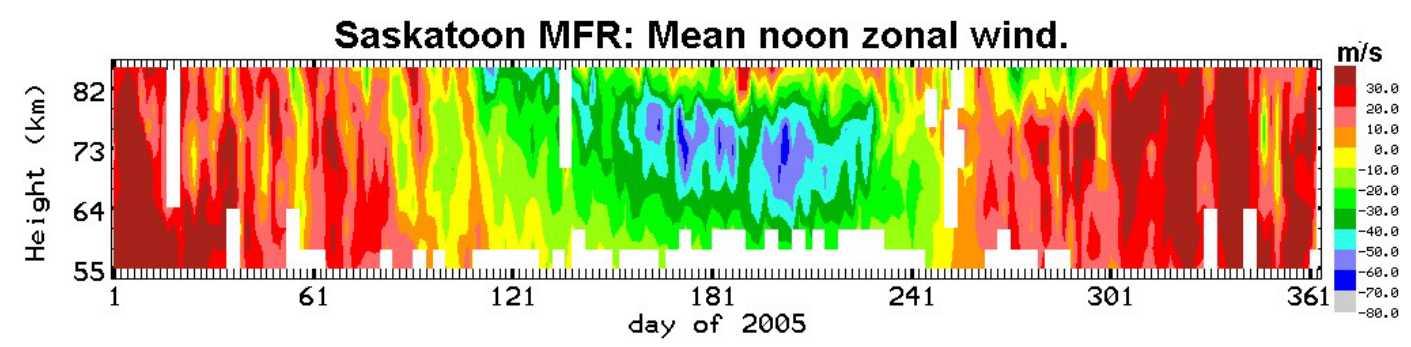

Fig. 2. Daily noon zonal wind at Saskatoon.

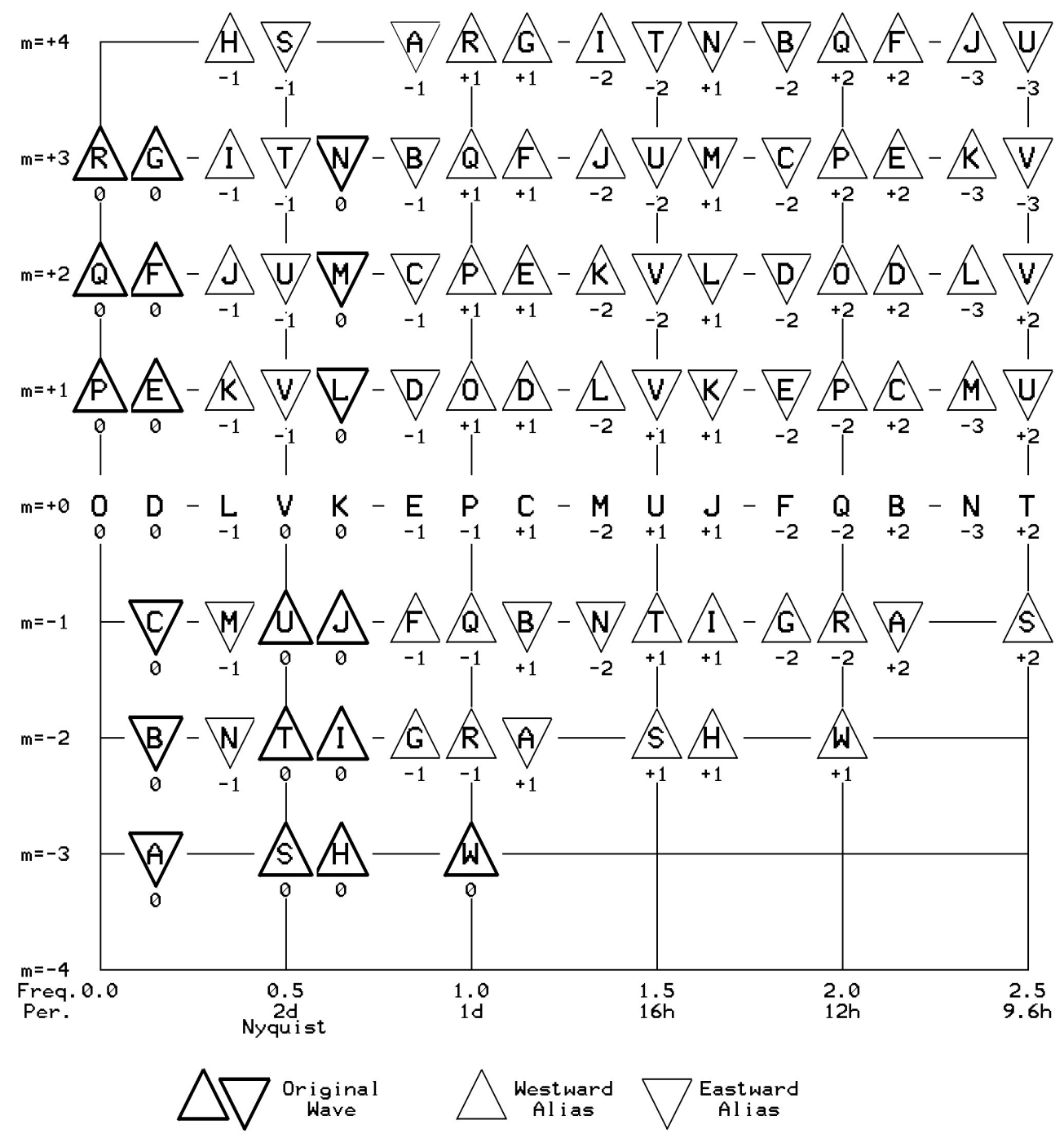

Fig. 3. Chart of aliases for a selection of original waves (see text for full explanation).

For long term intervals it must also be taken into account that SABER precesses $3^{\circ}$ per day eastward (i.e. faster than the earth turns), so $t_{s}=360 / 363 \mathrm{~d}$.

Figure 3 is a chart of original waves and some of their aliases (from Eq. 4). An alphabetic character surrounded by a heavy triangle is the original wave; the same character in light triangles are its aliases (converted if necessary to positive frequency by replacing $f$ by $-f$ and $m$ by $-m$ ). The orientation of the triangles shows westward/eastward propagation (of the lowest positive frequency alias), and the "alias order" (" $n$ " in Eq. 4) relative to the original wave appears below the triangle. These orders can be subtracted to find the 

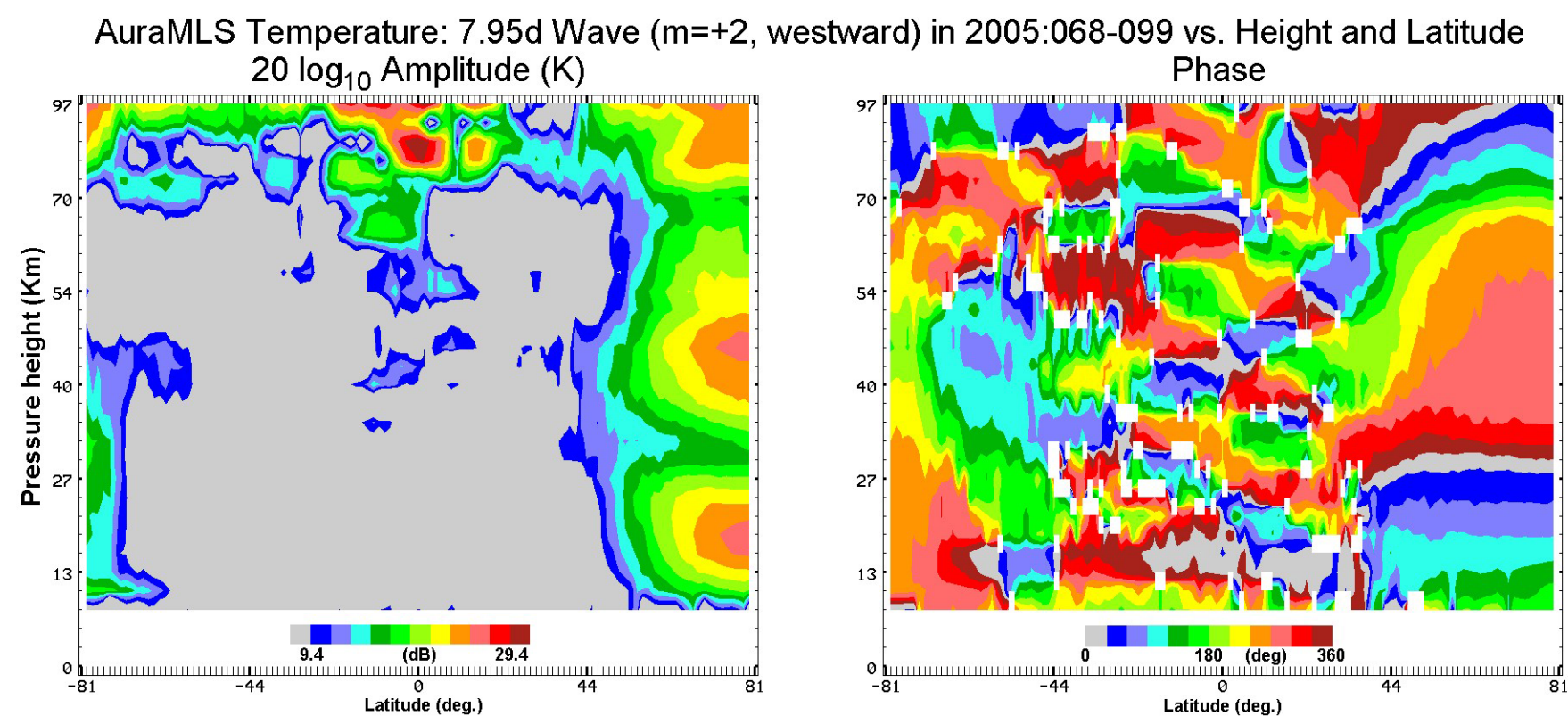

Fig. 4. Spring 2005 oscillation in temperature of period 7.95 d, wave number +2 : amplitude and phase values the Aura MLS. Data latitude range is: $-82 \mathrm{~S}$ to $82 \mathrm{~N}$.

"distance" between aliases for the purpose of finding relative amplitudes (viz. if one of aliases is considered to be the real wave). The frequency scale is in cycles per $t_{s}$. In the period scale $d$ should be replaced by $t_{s}$ for non-solar synchronous satellites. Of particular interest in winter are stationary wave aliases: for example wave 1 has an weak alias at $m=2,24 \mathrm{~h}$ at our latitude, and a strong one at $m=3,12 \mathrm{~h}$, among others (see Eq. 4, Table 1, and Fig. 1).

The foregoing model has assumed stationarity. In the real world wave events will have lifetimes. The wavelet plot is suited to this, but can be misleading while looking for aliases since a short burst at short period is not likely to lead to a long period alias, and also amplitudes, real or aliased, depend on the length of the real event relative to the window as well as on the alias order. This complicates matters considerably, to say the least.

On the other hand, near the poles, the satellite is moving fast in longitude and local time (the maximum speed is about 2 LT h, or 30 degrees of longitude, per minute), and some potential aliases may be averaged out by the effective sampling window, 1-2 min.

\subsection{Discussion of aliases in relation to the measure- ments}

As stated previously the "noise" level is expected to be higher at the higher frequencies because of the wider bandwidth for short sequences. This is likely obscuring many of expected aliases, but with reference to Fig. 1 , the peak at $m=-1$, period $\mathcal{T} \sim 5$ d, near day 31 would be like " $C$ " in Fig. 3 We expect to see it also at a period between $24 \mathrm{~h}$ and $2 \mathrm{~d}$ at $m=+2$, and there is a small feature there. The feature $m=3, \mathcal{T} \sim 5 \mathrm{~d}$ near day 345, like "G" in Fig. 3, should have an alias at $m=-2$ between $24 \mathrm{~h}$ and $2 \mathrm{~d}$. It does, and is stronger so we have to rule out the $m=3$ peak. There is also is a third possibility at $m=-1$.

\section{Selected PW, wave number $m=2$, period $T=7.95 \mathrm{~d}$}

The apparent planetary wave in Fig. 1, day $\sim 91$, at $m=+2$, period $\mathcal{T}$ of $7.95 \mathrm{~d}$ has been chosen for detailed analysis. According to Fig. 3 (wave "F") its nearest alias should be at $m=-1$, and $\mathcal{T}=1.14 \mathrm{~d}(27 \mathrm{~h})$ with a relative amplitude (Table 1 ) of 0.17 , viz. about $26 \mathrm{~dB}$ below the original. With the present plot scale in Fig. 1 this alias is at the lowest contour level, which is probably why it cannot be seen. A second order alias is at $m=+4$ (not shown in Fig. 1) and $\mathcal{T}=21 \mathrm{~h}$. This should have a relative amplitude of 0.94 , but it does not seem reasonable to expect a $21 \mathrm{~h}$ oscillation to remain coherent for a significant part of the $6 \mathcal{T}$ window where $\mathcal{T}=8 \mathrm{~d}$. Figure 4 shows the result of fitting, at each latitude and height, an $m=+2, \mathcal{T}=7.95 \mathrm{~d}$ wave to $32 \mathrm{~d}$ of temperature data centred on the time for maximum amplitude. The phase is in degrees relative to 00:00 UT on 1 January 2005. The amplitudes and phases show a propagating wave from from $80^{\circ} \mathrm{N}$ to about $45^{\circ} \mathrm{N}$ with a vertical wavelength of $\sim 60 \mathrm{~km}$ in the upper mesosphere; the repetitious amplitude peaks match with smaller vertical phase gradients, which are suggestive of variable propagation conditions in the atmosphere e.g. background winds and temperatures. Ground based measurements can be used to confirm whether the selected period 

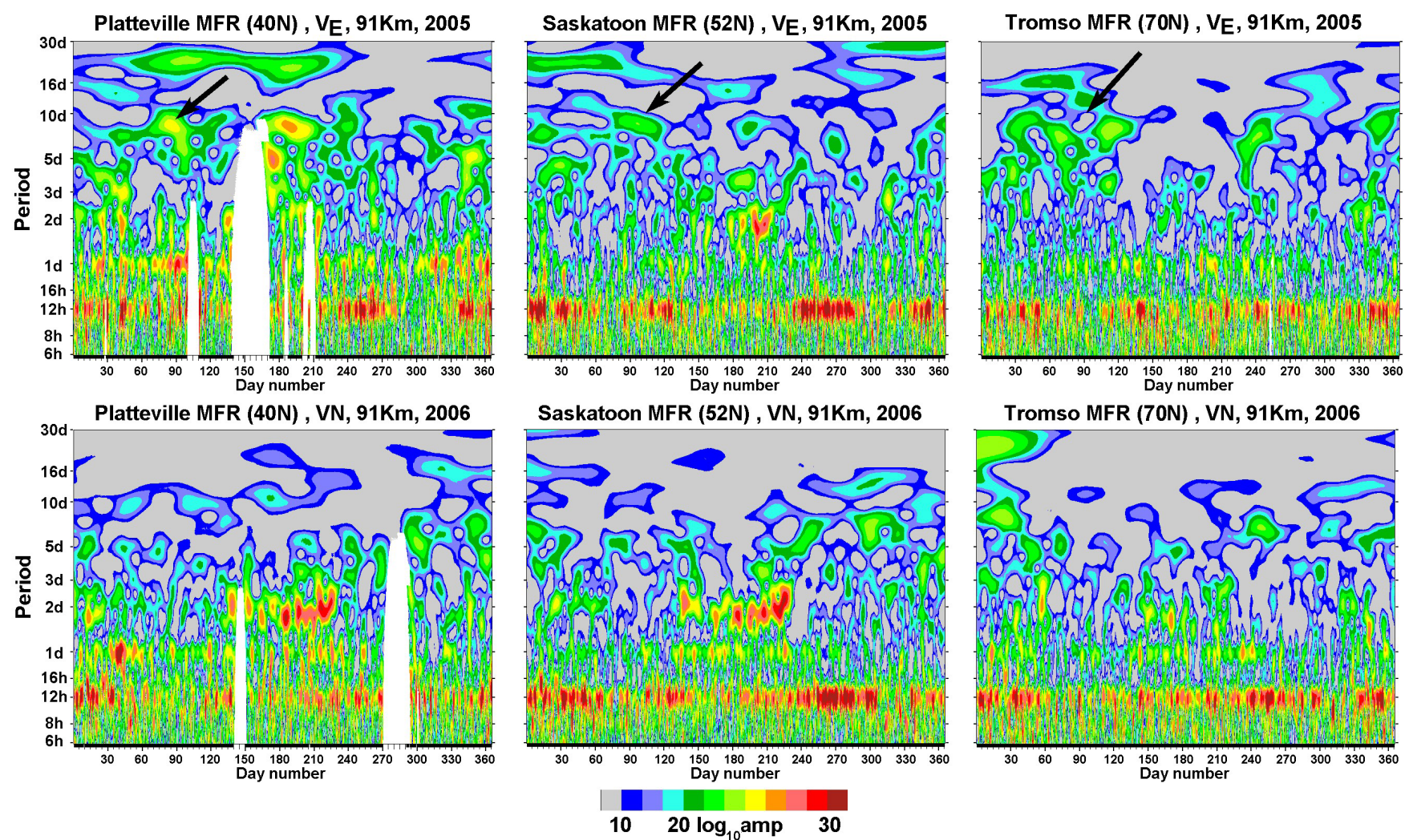

Fig. 5. Wavelets of zonal radar winds for 2005 at $91 \mathrm{~km}$ for Platteville $\left(40^{\circ} \mathrm{N}, 105^{\circ} \mathrm{W}\right)$, Saskatoon $\left(52^{\circ} \mathrm{N}, 107^{\circ} \mathrm{W}\right)$, and Troms $\varnothing\left(70^{\circ} \mathrm{N}\right.$, $\left.19^{\circ} \mathrm{E}\right)$. Overplotted arrows show where the $8 \mathrm{~d}$ wave should appear. The colour scale is $\mathrm{dB}\left(20 \log _{10}\right.$ Amp $)$. Meridional wind wavelets for 2006 are included.

is correct or just an alias of a higher frequency event. In this case we just have wind data, but since planetary waves affect both wind and temperature, the effort is still worthwhile, although the global PW structure could be such that the wind and temperature amplitudes peak at different latitudes. The latter appears to be the case for the three radar sites shown in Fig. 5, in which the selected wave in the east-west wind component is strongest at Platteville, weak at Saskatoon, and not present at $7.95 \mathrm{~d}$ at Troms $\varnothing$. Regarding the latter site, however, there is a peak near $7 \mathrm{~d}$ that could be a local manifestation of the $8 \mathrm{~d}$ wave of interest. The studies of Luo et al. (2002) show such changes in observed PW periods from Tromsø to Christmas Island, and discuss these in terms of possible Doppler shifting and/or changing resonance conditions in different hemispheric locations.

\section{Summer frequency "dish"}

We now return to the feature that was the main instigator for this study, the summer "dish" shape of amplitudes in frequency-time spectra seen in both satellite temperature (Fig. 1) and ground based radar winds. Though the latter is not clearly seen in the zonal wavelets for 2005 in Fig. 5, it is clearer for that figure's meridional wavelets of 2006, and it is obvious in long term averages. Garcia et al. (2005), using SABER temperature data, found $5 \mathrm{~d}$ periods in the equinoxes, and shorter periods during the summer months. They attribute this to "selective absorption/dissipation of the slower waves as they propagate upward." Figure 6 shows the average power over 17 yearly wavelet analyses for Saskatoon, in which the dish is especially clear in the north-south wind, which has the larger component-amplitudes for periods below 5 days. Oscillations with periods greater than $\sim 5 \mathrm{~d}$ are relatively very weak in summer-centred months, when compared with the winter and equinox months. The EW components of these longer period waves are larger than the NS components (Fig. 6), as first shown by Luo et al. (2002). Otherwise, their variable periods and occurrences over the 17 years leads to relatively low mean powers when compared with the coherent and regular oscillations associated with the Quasi 2 Day Wave (Q2DW) and the solar tides (e.g. Manson et al., 2006). Our argument is that the mid summer westward jet between 70 and $80 \mathrm{~km}$ (Fig. 2) will filter out/block waves with lower phase speeds, $c$, as they experience critical levels. As mentioned previously, this blocking or exclusion limit, which is based on the peak Saskatoon radar winds in the mesospheric region, was calculated and plotted as an overlay 


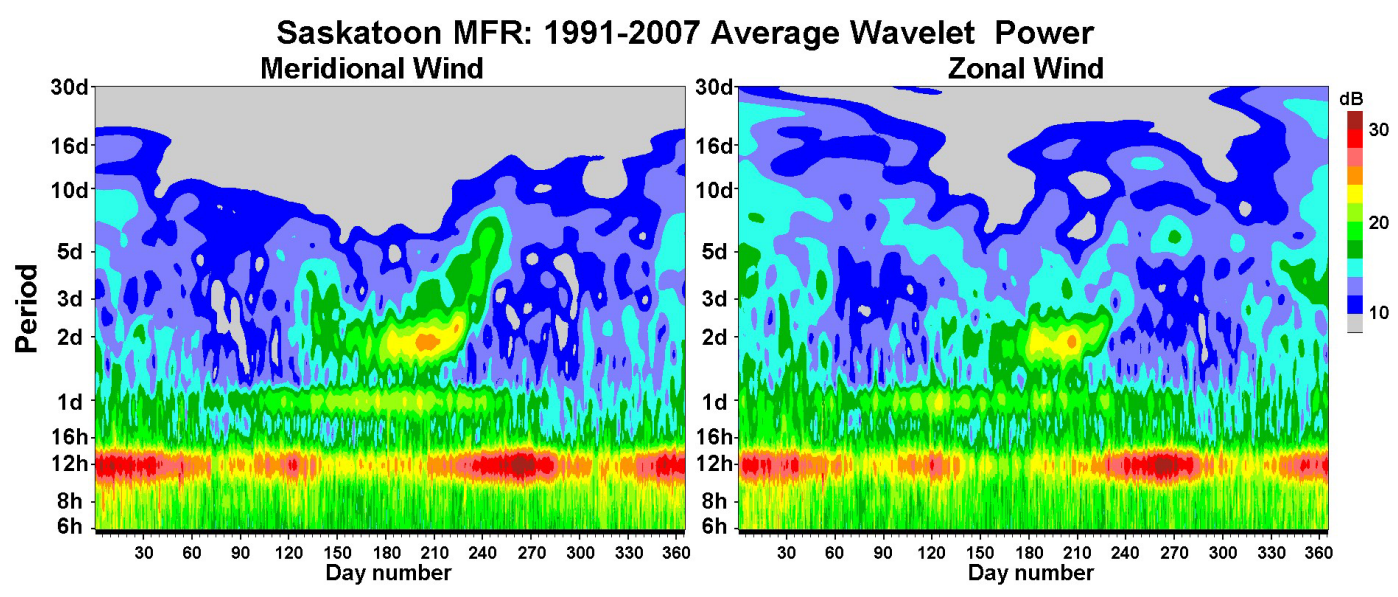

Fig. 6. A 17 year (1991-2007) average wavelet power plot for Saskatoon radar wind at $91 \mathrm{~km}$, meridional and zonal components.

in Fig. 1. It is a close but not a perfect match to the dish. One reason is that although the waves are from global temperature data, the calculated phase speed limit imposed by the zonal wind jet is based on one site (using maximum daily noon wind in the height range $58-79 \mathrm{~km}$, which had then been averaged over the same window as used in the wavelet calculation). Some waves in Fig. 1 are seen at $92 \mathrm{~km}$, particularly at $m=+3$ but also $m=+1$ and +2 , which according to theory should not have penetrated this jet. Notice again however the PW of longer period, viz. lower phase speed, further from the overplotted limit line, are excluded. It is possible that the wind value is biased because of tidal contamination, or because a single site cannot properly represent the global zonal wind.

Monthly fits of mean plus tides ( $24 \mathrm{~h}, 12 \mathrm{~h}$ ) were applied to Saskatoon radar data and showed that tidal contribution to the noon zonal values is of the order of $5-10 \mathrm{~m} / \mathrm{s}$ at $79 \mathrm{~km}$, and is usually positive. That is, the noon wind is more westward than the daily mean. However at lower heights, say $70 \mathrm{~km}$, where the wind can be strongest, the tidal contamination is smaller, and sometimes negative. There is also the issue as to the duration of any regime in background winds: this should be for at least as long as the range of PW periods in question.

More important is the variation of zonal wind with longitude. There appears to be a significant standing wave in summer. Reference to UKMO/MetO assimilated model winds at the $0.3 \mathrm{mb}$ level (circa $\sim 56 \mathrm{~km}$ ) shows that in 2005 the average July zonal wind speed has its maximum at $300^{\circ} \mathrm{E}$ $(\bar{U}=-61 \mathrm{~m} / \mathrm{s})$ and minimum at $130^{\circ} \mathrm{E}(\bar{U}=-42 \mathrm{~m} / \mathrm{s})$. A similar pattern was found in the two other years checked, 2003 and 2004, but it had slightly reduced maxima. That is, Saskatoon zonal winds are stronger than the global mean. If this pattern continues up to the jet maximum it could help to explain how some waves do not experience critical levels near $70 \mathrm{~km}$. For example in 2005 (Fig. 1), the maximum mid-summer (windowed) radar wind value used was $84 \mathrm{~m} / \mathrm{s}$, equivalent to a limiting period of $1.13 \mathrm{~d}$ (at $m=+3$, and $51.7^{\circ} \mathrm{N}$ ). If we scale by the MetO value ratio, on the assumption that some fraction of the wave energy can penetrate at the weakest wind location, the limit would be $1.64 \mathrm{~d}$.

An alternate explanation, suggested by a reviewer, is that gravity waves modulated by lower stratosphere wind might pass through the jet and deposit their energy at greater heights, thus re-generating the PW. This method was proposed by Smith (2003) for producing stationary planetary waves in the upper mesosphere.

It is interesting that the modulation in the mid-summer quasi two day temperature wave (Q2DTW), in the $m=+3$ sector of Fig. 1, in mid summer is similar to the modulation of the zonal wind.

The appearance depends partly upon the windowing, which is the same for both, but the impression is that the Q2DTW is absent for the strongest winds - that is for periods above the two lowest values of the overplotted phase speed limit. This result is consistent with the westward phase velocity being then consistently lower than the background wind and hence the occurrence of critical levels. More generally, the monthly occurrences of the Q2DW at $m=+3$, which include winter as well as summer months, are very consistent with the radar-wind studies of this wave (Meek et al., 1996; Chshyolkova et al., 2005). We also note that although the non-linear interaction between the Q2DW and the diurnal tide has been documented for particular events (Manson et al., 1998; Pancheva, 2006), instances of the production of $16 \mathrm{~h}$ oscillations are not numerous enough to lead to a spectral peak in Fig. 6.

Palo et al. (2007) show a proposed Q2DW - migrating diurnal tide interaction product, a weaker eastward propagating wave with period slightly less than $2 \mathrm{~d}$ found in SABER temperature data near $45^{\circ} \mathrm{S}$, The situation is comparable to "I" in Fig. 3, with the actual wave at $m=+3$, period a little greater than $2 \mathrm{~d}$, and an alias at $m=2$, with period a little less than $2 \mathrm{~d}$. If the expected strength of the alias is much weaker than that found, then non-linear interaction is a possible candidate. 
However for SABER at $45^{\circ} \mathrm{S}$ in the late January yaw configuration (maximum southern latitude sampled is $\sim 50^{\circ} \mathrm{S}$ ), the $\mathrm{A}$ and $\mathrm{D}$ samples are quite close together in longitude, and so the alias should be strong, similar to Aura at $80^{\circ} \mathrm{N}$. On the other hand, SABER's slow precession may have to be taken into account.

\section{PW wave number spectra and the "best mode"}

Firstly, a few general comments on Fig. 1 (and indeed Figs. 4 and 5) are appropriate. The dominance of periods $\mathcal{T}$ near 5, 10 and $16 \mathrm{~d}$ and with wave number $m=+1$ during months with eastward background winds is consistent with radar wind studies by Luo et al. (2002) and others: these are the socalled Rossby Waves. Other studies have noted oscillations in the temperature field with periods near 5 days and $m=+1$ during summer months at high latitudes (Kirkwood et al., 2002). There has been little discussion in the literature of observed PW with $m<0$, consistent with the smaller intensities of those wave numbers in Fig. 1, and the theoretical requirement for PW to have phase velocities that are westward with respect to the background wind (Andrews and Holton, 1987).

There is another limit associated with the propagation of PW into the middle atmosphere: the difference between the background wind and the PW phase speed (allowing for sign) shall be less that a so-called "critical velocity" $U_{c}$ (Charney and Drazin, 1961; Andrews and Holton, 1987; Manson et al., 2005). Thus in the winter months the Rossby Waves will propagate vertically more strongly when the eastward winds (westerlies) are weaker e.g. during the major stratospheric warmings, than when the winds associated with the winter polar vortex are at their maximum. Consistent with this, in Fig. 1 for $m=+1$, winter-PW with periods of less than 5 days that would have large phase speeds, are seldom in evidence (usually excluded); while oscillations of these periods are more evident at $m=+2$ and 3. This is particularly clear for the PW of period near 2 days and $m=+3$, which is the winter manifestation of the Rossby-gravity wave Q2DW. As discussed elsewhere (Nozawa et al., 2003; Chshyolkova et al., 2005) these waves had not been considered until recently, due to the radar-types used and emphasis upon the Summer Polar Mesospheric Echoes (PMSE) in the Scandinavian sector.

We return to Fig. 1: which spectral peaks represent actual PWs and which are their aliases? Since these latter will show generally smaller amplitudes, never bigger than the actual wave, one way to select is by amplitude. To do this we will borrow an idea from Manson et al. (2004a): different modes are coded with different colours, and the mode with maximum amplitude is plotted.

However not everything is a PW, so we would like to apply a criterion for significance. An argument that has been used by Scargle (1982) is as follows. Consider a white noise data sequence of $v$ equi-spaced points with variance

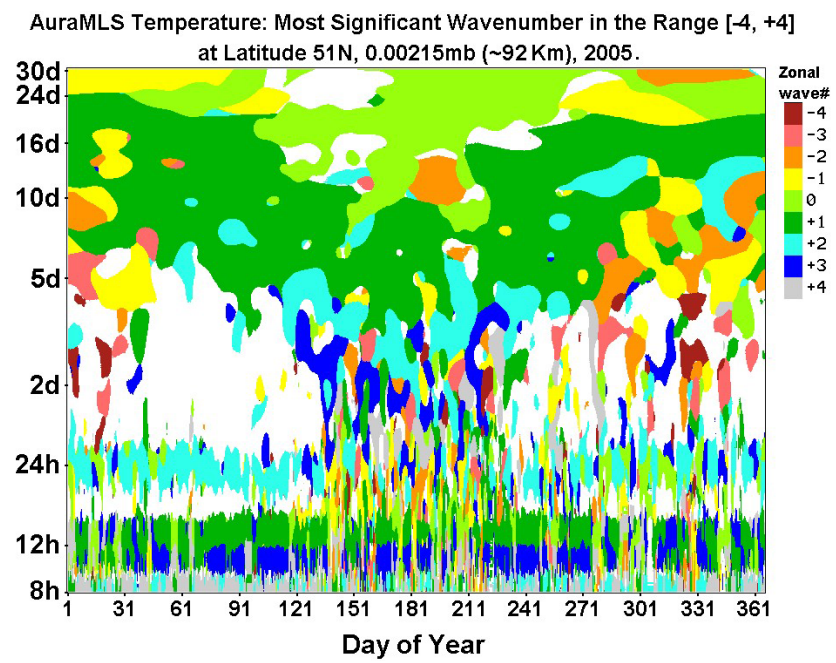

Fig. 7. A wavelet-format plot based on the analysis used in Fig. 1, but showing the wave number in the range -4 to +4 with greatest significance at each frequency/time.

$\sigma^{2}$. The expected power in a single spectral bin is then $P_{a v}=\sigma^{2} / \nu$ because power is equally distributed over all freqency bins, and the power in any bin $\mathrm{f}$ relative to the expected is $z=P(f) / P_{a v}$.

The Scargle assmption is that the white noise is such that $z$ has an exponential distribution, viz. the probability of finding a value of $z$ between $z$ and $z+d z$ is $e^{-z}$. Therefore the probability of finding $z<z_{c}$ in noise is $\left(1-e^{-z_{c}}\right)$. The corresponding signifiance level, viz. the percentage probability that the signal is not noise, is $100\left(1-\left[1-e^{-z_{c}}\right]\right)$.

The second argument used is that if a spectrum with $\mu$ independent frequencies is examined, there are more chances to find $z>z_{c}$ in white noise, and therefore the probability of $z<z_{c}$ is reduced to $\left(1-e^{-z_{c}}\right)^{\mu}$. Thus more cases of $z>z_{c}$ are expected, and the percentage significance level, that is the probability of $z<z_{c}$ is reduced to $100\left(1-\left[1-e^{-z_{c}}\right]^{\mu}\right)$.

In relation to the present analysis, it seems that $\mu$ is always one since, with a sequence 6 times the period, we are only looking at one frequency. However when the frequencytime plot is considered as a whole, the arguments about $\mu$ are more difficult. For the present we will use $\mu=1$ and apply a somewhat arbitrary significance level. $z_{c}$ will be the ratio of individual frequency power to the part of the total sequence variance we would expect to find at that frequency if the data were pink noise with a log-frequency log-power slope of -1 to -2 . (The actual \% level used was arbitrarily chosen to clear out some of the weaker areas of the next figure, 7.) As mentioned before, if we use this significance scheme and increase the level, the last remaining data are at 12 and $24 \mathrm{~h}$.

Figure 7 shows the mode with the highest amplitude (and passing the previously mentioned ad hoc significance level) at each wavelet period versus time (day of the year). We have added wave number $m=4$ to the analysis. The "layering" of 
wave numbers is interesting. It seems that PW of increasingly low period/high frequency possess higher wave numbers and vice versa, even though we have previously argued that the jet should usually filter out waves with the combination of period/frequency and mode of slower moving waves (i.e. PW with larger wave numbers at a given period.) This filtering process certainly applies for $\mathcal{T}>10 \mathrm{~d}$ and $m=+1$ in Fig. 1 , as well as $\mathcal{T}>5 \mathrm{~d}$ for $m=+2$ and $\mathcal{T}>3 \mathrm{~d}$ for $m=+3$. However we find here that the waves of highest intensity (Figs. 1 and 7) at low frequencies, e.g. 5-10 d, in mid summer are preferably still $m=1$, although their estimated phase speeds are less than that of the jet; PW in this spectral range but with $m=+2$ would have even smaller phase speeds and experience a critical level at even lower altitudes.

This concern regarding Fig. 7 is similar to that discussed in Sect. 6 with regard to Fig. 1, where it was noted that the longitudinal maximum zonal wind could be lower than that used to produce the over-plotted line representing the critical level for the PW oscillations. There it was noted that the zonal mean speeds could well be less than at Saskatoon, and hence the over-plotted line could be located at periods during mid summer which are somewhat low. Alternate possibilities are some PW leakage or penetration at the critical level; that there is atmospheric resonance at these preferred Rossby wave spatial and temporal scales; that PW of these periods, viz. waves with low frequency and higher wave number, do not exist; or as a refereee has suggested, that they may have propagated from the other hemisphere.

Certainly, and in summary, it is the case that Rossby waves of periods near 10 and 16 days are rationally excluded from the summer atmosphere using the critical level argument and the associated over-plotted line; that the preferred/dominant wave number for PW of periods equal to or greater than 5 days is $m=1$; that PW periods between 2 and 5 days are dominantly $m=+2$, and that for the Q2DTW $m=+3$ is statistically favoured in the temperature field at middle latitudes.

\section{Summary and conclusions}

We have shown that the likely explanation for the "dish" shape in summer-centred frequency-time spectra at midlatitude is that planetary waves (PW) with sources in the lower atmosphere propagate into the mesosphere during the winter and neighboring equinoctial months, but that systematically those with phase speeds lower than that of the summer-like zonal stratospheric-mesospheric jet at 50$80 \mathrm{~km}$, are filtered out or blocked from entering the upper mesosphere. The "critical level" overplotted on Fig. 1, where the PW zonal phase speed matches the background flow, has a seasonal variation in the spring and fall months that parallels that of the reduction of PW activity with long periods (10-16 days). These long periods dominate the winter centred months of the upper mesosphere, when a similar phase speed limit does not exist. In the middle of summer the re- maining mesospheric $\mathrm{PW}$, with periods of 2-5 days and wave numbers of +3 to +1 respectively, are clustered close to the critical level position.

A detailed discussion of satellite frequency-wave number aliases resulted in a "look-up chart" and an "expected amplitude" table. Mathematically these aliases will always exist, and at the predicted amplitude relative to the actual wave. In our opinion, the only internal way to distinguish actual versus aliased waves is by their relative amplitudes and by physical arguments about their likelihood.

The inspection of spectra from ground-based monitors is valuable in settling the frequency alias question, but in this case, where satellite and radar measure different parameters, it is not an ideal method. Here a selected temperature wave was seen increasing in amplitude and stable in phase from 45 to $82^{\circ} \mathrm{N}$, but in wind measurements the strongest manifestation was at Platteville $\left(40^{\circ} \mathrm{N}\right)$. It was weaker at Saskatoon $\left(52^{\circ} \mathrm{N}\right)$ and not seen at all, or overshadowed by local effects, at Troms $\varnothing\left(70^{\circ} \mathrm{N}\right)$. Such latitudinal variations are not unusual, given the observations of the $5 \mathrm{~d}$ period $\mathrm{PW}$ in the temperature field of northern Scandinavia, and its relative absence at middle latitudes such as Saskatoon.

\section{Appendix A}

In the following identification of aliases we will assume regular sampling - viz. constant time/longitude steps at any latitude. We do not consider wavenumber aliases, which for Aura implies wavenumbers greater than 7 , on the argument that such a small spatial feature unlikely to be globally coherent. A simple place to start is one-node sampling; e.g. the EP TOMS satellite, where sample longitude is directly related to sample time. However we will not necessarily assume sun synchronicity as we do for TOMS or Aura. Time $t_{s}$ will denote the time for the satellite to cover all longitudes (frequency $f_{s}$ ) and $t_{\text {orb }}$ the time for an orbit. For example Aura has $t_{s}=1 \mathrm{~d}$ and $t_{\text {orb }} \sim 90 \mathrm{~min}$. The (temporal) Nyquist frequency for single node sampling is $f_{s} / 2$. This is easy to see if it is imagined that orbital period is such that the same longitudes are sampled each day.

As in Eq. (1) a specific planetary wave at a given latitude and height in some parameter, $v$, can be written

$v=V_{0} \cos \left(\omega_{1} t+m_{1} \ell-\phi_{1}\right)$

The sample times $t$ are discrete and equi-spaced and the sample longitudes are related to them by $\ell=-2 \pi t / t_{s}$ plus a constant ( the earth is turning "Eastward" under the satellite)

The result of a "FT" at an arbitrarily chosen frequency $f$ and wavenumber $m$ is

$$
\begin{aligned}
X_{R} & =\frac{1}{n} \sum_{1}^{n}\left\{V_{0} \cos \left(\omega_{1} t+m_{1} \ell-\phi_{1}\right) \cos (\omega t+m \ell)\right\} \\
& =\frac{1}{n} \sum_{1}^{n}\left\{V_{0} \cos \phi_{1} \cos \left(\omega_{1} t+m_{1} \ell\right) \cos (\omega t+m \ell)\right.
\end{aligned}
$$




$$
\left.+V_{0} \sin \phi_{1} \sin \left(\omega_{1} t+m_{1} \ell\right) \cos (\omega t+m \ell)\right\}
$$

$$
\begin{array}{r}
X_{I}=\frac{1}{n} \sum_{1}^{n}\left\{V_{0} \cos \left(\omega_{1} t+m_{1} \ell-\phi_{1}\right) \sin (\omega t+m \ell)\right\} \\
=\frac{1}{n} \sum_{1}^{n}\left\{V_{0} \cos \phi_{1} \cos \left(\omega_{1} t+m_{1} \ell\right) \sin (\omega t+m \ell)\right. \\
\left.\quad+V_{0} \sin \phi_{1} \sin \left(\omega_{1} t+m_{1} \ell\right) \sin (\omega t+m \ell)\right\}
\end{array}
$$

For $X_{R}$ or $X_{I}$ to have a non zero result, $\overline{\cos x \cos y}$ or $\overline{\sin x \sin y}$ must have $x=y$ or $x=y \pm \pi$, and $\overline{\sin x \cos y}$ must have $x=y \pm \pi / 4$ (thus converting at least one product to $\pm \sin \sin$ or $\pm \cos \cos )$.

Any non zero average in this "monochromatic", infinite data length, case represents an alias; so a real wave with parameters $\omega_{1}, m_{1}$ has aliases given by:

$\omega_{1} t+m_{1} \ell=\omega t+m \ell \pm n \pi / 4$

where $m$ must be an integer, and $n=0,1, \ldots$ because each case results in at least one sin sin or cos cos term. However in practice the additional phase term, $\varphi=n \pi / 4$ can be shown to be zero as follows. In

$\omega_{1} t+m_{1} \ell=\omega t+m \ell+\varphi$

we know that in sampling at one latitude circle $\ell$ and $t$ are related by $\ell=-2 \pi t / t_{s}$. This means that $\varphi$ must also be a function of $t$, therefore the only constant it can be is zero.

So finally the only aliases to $m_{1}, f_{1}$ are

$m=m_{1}+n, \quad \frac{f}{f_{s}}=\frac{f_{1}}{f_{s}}+n$

where $m, m_{1}$ must be integers, and $n=0, \pm 1, \pm 2, \pm 3 \ldots$

When only one node is used, all aliases have the same amplitude.

Two node sampling is a more interesting case. Each node has the same alias frequencies, because the sampling is equivalent except for a constant longitude offset, but potentially different phases for the A and D aliases, which may result in some cancellation. The Nyquist remains $f_{s} / 2$ for each node.

Let the time and longitude for ascending node samples be $t_{A}$ and $\ell_{A}$, and for descending node be, $t_{D}$ and $\ell_{D}$. Then

$v_{A}=V_{0} \cos \left(w_{1} t_{A}+m_{1} \ell_{A}-\phi_{A}\right)$

and

$v_{D}=V_{0} \cos \left(w_{1} t_{D}+m_{1} \ell_{D}-\phi_{D}\right)$

For a fixed latitude, $t_{A}-t_{D}$ and $\ell_{A}-\ell_{D}$ are constants. The spacing between $\ell_{A}$ and $\ell_{D}$ will vary with latitude, being close to $\pi$ at the equator (the earth has turned by $\pi t / t_{\text {orb }}$ radians between samples at the equator) and 0 at the North or South-most sample point. This can be calculated from the tilt of the orbit, $\gamma$, and the latitude, $\alpha$. The two sample points are spaced by an "azimuth" angle

$\Delta L=2 \cos ^{-1}(\tan \alpha \tan \gamma)$

radians in "longitude", but since the samples aren't simultaneous, and the earth has rotated somewhat between samples, this is not the difference in longitude between samples (unless it is imagined that the earth is not rotating.)

Therefore the result of using A and D data (with the FT analysis) is equivalent to adding the wave vectors for $\mathrm{A}$ and $\mathrm{D} ; V_{0}$ will be the same for separate $\mathrm{A}$ and $\mathrm{D}$ aliases but the $\mathrm{A}$ and $\mathrm{D}$ phase difference effects some cancellation.

$\mathrm{Amp}=\sqrt{\left(V_{0} \cos \phi_{A}+V_{0} \cos \phi_{D}\right)^{2}+\left(V_{0} \sin \phi_{A}+V_{0} \sin \phi_{D}\right)^{2}}$

For the "real" wave, $\phi_{A}=\phi_{D}$, so the correct amplitude is obtained. If these phases are not equal some, or maybe major, cancellation will result. It can be shown that the wave amplitude is given by:

$V_{A \& D}=V_{0} \cos \frac{n}{2} \Delta L$

where $V_{0}$ is the amplitude of the actual wave $(n=0)$ and $n$ is the "alias order": $0, \pm 1, \pm 2, \ldots$ A (pleasant) surprise is that there is no dependence on real wave mode or frequency, just on the order of the alias.

Acknowledgements. The authors are grateful to the Jet Propulsion Lab (JPL) for access to the Aura MLS data. Due to superior archiving, it is very nice data to work with! We are also grateful to the British Atmospheric Data Centre (BADC) for the stratospheric assimilated model (UKMO/METO), to the Institute of Space and Atmospheric studies, through the University of Saskatchewan, for research facilities, and to Canada's National Sciences and Engineering Research Council (NSERC) for financial support through a Discovery Grant. We appreciate the careful assessment and suggestions by the two reviewers.

Topical Editor C. Jacobi thanks two anonymous referees for their help in evaluating this paper.

\section{References}

Andrews D. G., Holton, J. R., and Leovy, C. B.: Middle Atmosphere Dynamics, Academic Press (San Diego), pp. xi+489, 1987.

Charney, J. G. and Drazin, P. G.: Propagation of planetary-scale disturbances from lower into the upper atmosphere, J. Geophys. Res., 66, 83-109, 1961.

Chshyolkova, T., Manson, A. H., and Meek, C. E.: Climatology of the quasi two-day wave over Saskatoon $\left(52^{\circ} \mathrm{N}, 107^{\circ} \mathrm{W}\right): 14$ years of MF radar observations, Adv. Space Res., 35(11), 2011 2016, 2005.

Garcia, R. R., Lieberman R., Russell III, J. R., and Mylnczac, M. G.: Large-scale waves in the mesosphere and lower thermosphere observed by SABER, J. Atmos. Sci., 62, 4384-4399, doi:10.1175/JAS3612.1, 2005. 
Goupillaud, P., Grossman, A., and Morlet, J.: Cycle-octave and related transforms in seismic signal analysis, Geoexploration, 23, 85-102, 1984.

Hall C. M., Aso, T., Manson, A. H., Meek, C. E., Nozawa, S., and Tsutsumi, M.: High-latitude mesospheric mean winds: A comparison between Troms $\varnothing\left(69^{\circ} \mathrm{N}\right)$ and Svalbard $\left(78^{\circ} \mathrm{N}\right)$, J. Geophys. Res., 108(D19), 4598, doi:10.1029/2003JD003509, 2003.

Kirkwood S., Barabash, V., Brändström, B. U. E., Moström, A., Stebel, K., Mitchell, N., and Hocking, W.: Noctilucent clouds, PMSE and 5-day planetary waves: A case study, Geophys. Res. Lett., 29(10), 1411, doi:10.1029/2001GLO14022, 2002.

Luo, Y., Manson, A. H., Meek, C. E., Meyer, C. K., Burrage, M. D., Fritts, D. C., Hall, C. M., Hocking, W. K., MacDougall, J., Riggin, D. M., and Vincent, R. A.: The 16-day planetary waves: multi-MF radar observations from the arctic to equator and comparisons with the HRDI measurements and the GSWM modelling results, Ann. Geophys., 20, 691-709, 2002, http://www.ann-geophys.net/20/691/2002/.

Manson, A. H., Meek, C. E., and Hall, G. E.: Correlations of gravity waves and tides in the mesosphere over Saskatoon, J. Atmos. Sol.-Terr. Phys., 60, 1089-1107,1998.

Manson, A. H., Meek, C. E., Avery, S. K., and Thorsen, D.: Ionospheric and dynamical characteristics of the mesosphere-lower thermosphere region over Platteville $\left(40^{\circ} \mathrm{N}, 105^{\circ} \mathrm{W}\right)$ and comparisons with the region over Saskatoon $\left(52^{\circ} \mathrm{N}, 107^{\circ} \mathrm{W}\right)$, J. Geophys. Res., 108(D13), 4398, doi:10.1029/2002JD002835, 2003.

Manson, A. H., Meek, C. E., Chshyolkova, T., Avery, S. K., Thorsen, D., MacDougall, J. W., Hocking, W., Murayama, Y., Igarashi, K., Namboothiri, S. P., and Kishore, P.: Longitudinal and latitudinal variations in dynamic characteristics of the MLT (70-95 km): a study involving the CUJO network, Ann. Geophys., 22, 347-365, 2004a, http://www.ann-geophys.net/22/347/2004/.

Manson, A. H., Meek, C. E., Hall, C. M., Nozawa, S., Mitchell, N. J., Pancheva, D., Singer, W., and Hoffmann, P.: Mesopause dynamics from the scandinavian triangle of radars within the PSMOS-DATAR Project, Ann. Geophys., 22, 367-386, 2004b, http://www.ann-geophys.net/22/367/2004/.

Manson, A. H., Meek, C. E., Chshyolkova, T., Avery, S. K., Thorsen, D., MacDougall, J. W., Hocking, W., Murayama, Y., and Igarashi, K.: Wave activity (planetary, tidal) throughout the middle atmosphere $(20-100 \mathrm{~km})$ over the CUJO network: Satellite (TOMS) and Medium Frequency (MF) radar observations, Ann. Geophys., 23, 305-323, 2005,

http://www.ann-geophys.net/23/305/2005/.
Manson, A. H., Meek, C., Chshyolkova, T., McLandress, C., Avery, S. K., Fritts, D. C., Hall, C. M., Hocking, W. K., Igarashi, K., MacDougall, J. W., Murayama, Y., Riggin, C., Thorsen, D., and Vincent, R. A.: Winter warmings, tides and planetary waves: comparisions between CMAM (with interactive chemistry) and MFR-MetO observations and data, Ann. Geophys., 24, 24932518, 2006, http://www.ann-geophys.net/24/2493/2006/.

Meek, C. E., Manson, A. H., Franke, S. J., Singer, W., Hoffmann, P., Clark, R. R., Tsuda, T., Nakamura, T., Tsutsumi, M., Hagan, M., Fritts, D. C., Isler, J., and Portnyagin, Y. I.: Global Study of Northern Hemisphere Quasi-2-Day Wave Events in the Summers of 1992 and 1991, J. Atmos. Terr. Phys., 58(13), 14011411, 1996.

Meyer, C. K. and Forbes, J. M.: Natural oscillations of the ionosphere-thermosphere-mesosphere (ITM) system, J. Atmos. Sol.-Terr. Phys., 59, 2185-2201, 1997.

Nozawa, S., Imaida, S., Brekke, A., Hall, C. M., Manson, A., Meek, C., Oyama, S., Dobashi, K., and Fujii, R.: The quasi 2-day wave observed in the polar mesosphere. J. Geophys. Res., 108(D2), 4039, doi:10.1029/2002JD002440, 2003.

Palo, S. E., Forbes, J. M., ZHang, X., Russell III, J. M., and Mlynczak, M.G.: An eastward propagating two-day wave: Evidence for nonlinear planetary wave coupling in the mesosphere and lower thermosphere, Geophys. Res. Lett., 34, L07807, doi:10.1029/2006GL027728, 2007.

Pancheva, D. V.: Quasi-2-day wave and tidal variability observed over Ascension Island during January/February 2003, J. Atmos. Sol.-Terr. Phys., 68, 390-407, 2006.

Salby, M. L.: Sampling theory for asynoptic satellite observations. Part I: Space-time spectra, resolution and aliasing, J. Atmos. Sci., 39, 2577-2600, 1982.

Schwartz, M. J., Lambert, A., Manney, G. L., Read, W. G., Livesey, N. J., Froidevaux, L., Ao, C. O., Bernath, P. F., Boone, C. D., Cofield, R. E., Daffer, W. H., Drouin B. J., Fetzer, E. J., Fuller, R. A., Jarnot, R. F., Jiang, J. H., Jiang, Y. B., Knosp, B. W., Krüger, K., Li, J.-L. F., Mlynczak, M. G., Pawson, S., Russell III, J. M., Santee, M. L., Snyder, W. V., Stek, P. C., Thurstans, R. P., Tompkins, A. M., Wagner, P. A., Walker, K. A., Waters, J. W., and Wu, D. L.: Validation of the Aura Microwave Limb Sounder temperature and geopotential height measurements, J. Geophys. Res., 113, D15S11, doi:10.1029/2007/JD008783, 2008.

Scargle, J. D.: Statistical aspects of spectral analysis of unevenly spaced data, Astrophys. J., 263, 835-853, 1982.

Smith, A. K.: The origin of stationary planetary waves in the upper mesosphere, J. Atmos. Sci., 60, 3011-3041, 2003. 